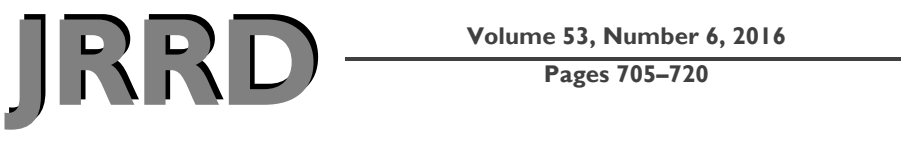

\title{
Chronic effects of exposure to high-intensity blasts: Results of tests of central auditory processing
}

\author{
Frederick J. Gallun, PhD; ${ }^{1-2^{*}}$ M. Samantha Lewis, PhD; ${ }^{1-2}$ Robert L. Folmer, PhD; ${ }^{1-2}$ Michele Hutter, MS; \\ Melissa A. Papesh, PhD; ${ }^{1}$ Heather Belding, BS; ${ }^{1}$ Marjorie R. Leek, PhD $^{1-3}$ \\ ${ }^{1}$ National Center for Rehabilitative Auditory Research, Rehabilitation Research \& Development Service, Department \\ of Veterans Affairs (VA) Portland Health Care System, Portland, OR; ${ }^{2}$ Department of Otolaryngology/Head \& Neck \\ Surgery, Oregon Health \& Science University, Portland, OR; ${ }^{3}$ VA Loma Linda Healthcare System and Department of \\ Otolaryngology/Head \& Neck Surgery, Loma Linda University Healthcare, Loma Linda, CA
}

\begin{abstract}
Patients who have recently experienced highintensity blasts are more likely to perform abnormally on tests developed to be sensitive to deficits of central auditory processing than were age- and hearing-matched individuals without blast exposures. Here, a group of 59 participants was recruited, 30 of whom were exposed to high-intensity blasts between 4 and $11 \mathrm{yr}$ prior to testing and did not participate in the previous study, along with 29 controls similar in age and hearing thresholds to the blast group. All were tested on a set of behavioral tests that were used in the previous study. Abnormal performance was measured with reference both to published normative data and to the average performance of the control group. Members of the blast-exposed group were again found to be significantly more likely to perform in the abnormal range than were the members of the control group. Because the patients in this study were tested a minimum of $4 \mathrm{yr}$ after blast exposure, these results suggest that for some of those exposed, problems processing auditory information may be a chronic effect of blast exposure even in the absence of significant peripheral hearing loss.
\end{abstract}

Key words: auditory dysfunction, auditory processing disorder, blast, central auditory processing, dichotic listening, hearing loss, psychoacoustics, rehabilitation, temporal processing, traumatic brain injury, Veterans.

Clinical Trial Registration: ClinicalTrials.gov: "Central auditory processing deficits associated with blast exposure"; NCT01567020; https://clinicaltrials.gov/ct2/show/NCT01567020

\section{INTRODUCTION}

For well over a decade, the U.S. military has been engaged in conflicts that have changed the nature of injury and survival among Veterans because of changes in weaponry, battle armor, and medical technology that have

Abbreviations: ANOVA $=$ analysis of variance; DDT $=$ Dichotic Digits Test; FPT = Frequency Patterns Test; GIN = GapsIn-Noise; HHIA = Hearing Handicap Inventory for Adults; $\mathrm{HL}=$ hearing level; ICD-9-CM = International Classification of Diseases, Ninth Revision, Clinical Modification; IOM = Institute of Medicine; MLD = Masking Level Difference; $\mathrm{mTBI}=$ mild traumatic brain injury; $\mathrm{N}_{0} \mathrm{~S}_{0}=$ noise in phase, signal in phase; $\mathrm{N}_{0} \mathrm{~S}_{\pi}=$ noise in phase, signal out of phase; PTA = pure tone average; PTSD = posttraumatic stress disorder; RR\&D = Rehabilitation Research \& Development; SRT = speech reception threshold; SSW $=$ Staggered Spondaic Words; VA = Department of Veterans Affairs; VAPORHCS = VA Portland Health Care System; VISN = Veterans Integrated Service Network; WARCAT $=$ Warrior Administered Retrospective Casualty Assessment Tool; WRS = word recognition score.

*Address all correspondence to Frederick J. Gallun, PhD; VA RR\&D National Center for Rehabilitative Auditory Research (NCRAR), 3710 SW US Veterans Hospital Rd, Portland, OR 97239; 503-220-8262, ext 57472; fax: 503721-1402. Email: Frederick.Gallun@va.gov http://dx.doi.org/10.1682/JRRD.2014.12.0313 
taken place over the course of the past $20 \mathrm{yr}$ [1]. According to the Institute of Medicine (IOM), these changes have resulted in a ratio of wounded to dead soldiers of 9.7 to 1 for the Global War on Terror, in contrast to rates of 4.3 to 1 or lower for all wars preceding it in the history of the United States [1]. Both the increased reliance upon explosives and the higher survival rate have created a landscape of new questions that must be answered in order to provide the best medical care for Veterans. This is true for hearing healthcare as much as for any other area of medicine. In 2014, the IOM issued a report on the chronic effects of exposure to high-intensity blasts [1]. In a systematic review of the literature, the IOM report found that symptoms of hearing loss are often present following blast exposure [2-3]. The report went on to state that while peripheral auditory dysfunction is likely to persist even after other effects have diminished, there was "inadequate" evidence in the literature to answer the question of whether or not central auditory effects persist beyond a period of 6 mo after blast exposure. The current report was motivated by ongoing research in our laboratory that has revealed evidence of an association between remote high-intensity blast exposure and the ability to process auditory information.

Previous reports have described the ways in which blast exposure could [4] and does [3] cause changes in the ability of listeners to perform on tests of central auditory processing measured within a year of blast exposure. Tasks upon which blast-exposed participants were most likely to perform poorly included those relying upon temporal pattern perception, auditory temporal resolution, binaural processing, and dichotic listening. In addition, it has been demonstrated [3] that these problems occur even when traditional audiometric test results and electrophysiological measures primarily assessing the auditory brainstem remain largely in the normal range. Furthermore, electrophysiological measures evaluating cortical function indicate that some of these same listeners show delayed and reduced peak amplitudes relative to the responses expected from young listeners with normal peripheral auditory function. This pattern is consistent with the hypothesis that blast exposure can cause damage to cortical areas responsible for auditory processing, as well as potentially damaging the connections among central auditory processing areas. Clinically, these patterns of dysfunction are unusual in younger listeners but have similarities with patterns of abnormal performance sometimes observed in older listeners.
Data collection and recruitment are currently ongoing, but this report has been compiled in order to provide timely information to the healthcare community regarding the question posed by the IOM concerning the possible persistence of the effects of blast exposure on the ability to process auditory information. To address this question, the behavioral data collected so far will be presented in a manner allowing direct comparison to the behavioral data from the previous study [3] of more recently blast-exposed patients. Additional measures not reported here, including a range of electrophysiological tests and a number of behavioral tests with speech and nonspeech stimuli, will be reported separately once the full cohort of participants has been recruited and tested.

\section{METHODS}

\section{Participants}

The goal of the study was to test blast-exposed Veterans, but no database of such Veterans exists and the military records that might help to identify these Veterans are not available through the Department of Veterans Affairs (VA) electronic medical record system. To overcome this fundamental limitation in our ability to identify these Veterans, participants were recruited based on a selfreported history of exposure to high-intensity explosions (loosely defined) over the $10 \mathrm{yr}$ prior to the start of the study. While it was anticipated that recruiting recent Veterans with a diagnosis of mild traumatic brain injury (mTBI) would be a more efficient method of identifying such participants than recruiting from the general Veteran population, an mTBI diagnosis was not required for inclusion in the blast-exposed group. This was justified both by the fact that mTBI diagnosis is based on symptoms other than auditory dysfunction and the earlier finding [3] that an mTBI diagnosis (or lack thereof) was much less predictive of auditory dysfunction than was self-report of exposure to a high-intensity blast. To that end, each of the study participants was recruited from the VA Portland Health Care System (VAPORHCS) through flyers posted at the VAPORHCS, contacting previous participants from studies at the National Center for Rehabilitative Auditory Research located at the VAPORHCS who had asked to be contacted for future studies, or through letters sent to Veterans enrolled at the VAPORHCS with diagnoses in their medical records indicating a history of mTBI. 


\section{Group Definitions}

\section{Blast Group}

Individuals were recruited who indicated that during military service they had been exposed to one or more high-intensity blasts with at least one occurring in the $10 \mathrm{yr}$ prior to the start of the study in 2011. As indicated in the "Intake Assessments" section, these reports were carefully examined to ensure that the event described qualified as being within $50 \mathrm{~m}$ of a large explosion. Those having mTBI diagnosis without blast exposure were excluded from this analysis. Thirty self-reported blastexposed Veterans completed testing on the behavioral experiments described here. Three blast-exposed Veterans withdrew with incomplete data, and two additional Veterans were excluded from the analysis because of questions regarding data integrity based on inconsistent responding. The ages and sex distributions of those 30 participants for whom results will be reported are shown in Table 1. Data regarding the details of blast exposure and diagnosis of mTBI are provided in Table 2.

Table 1.

Audiometric and demographical information on participants by group (control vs blast-exposed).

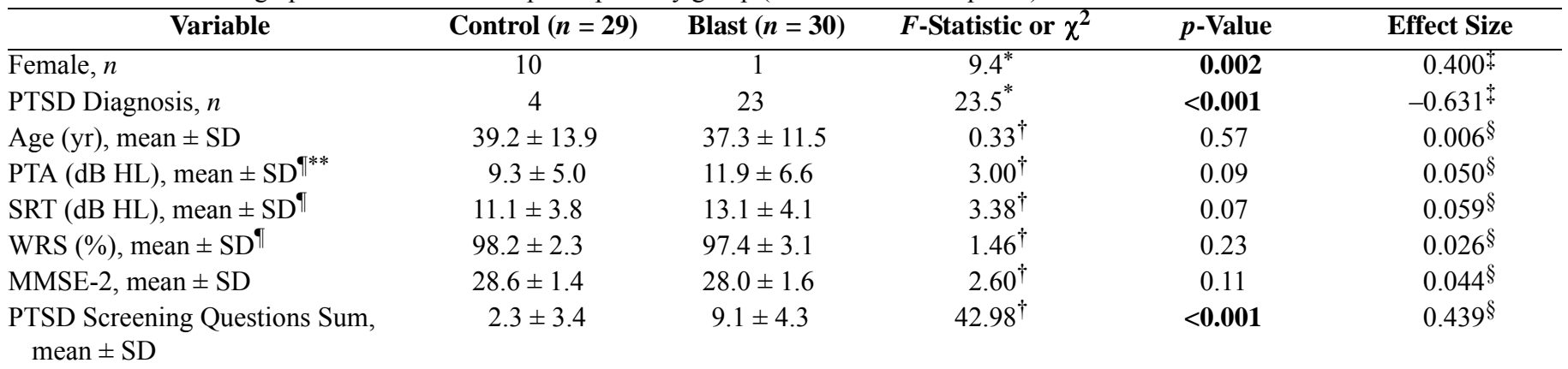

Note: Significant relationships $(p<0.05)$ are shown in bold.

${ }^{*} \chi^{2}$.

${ }^{\dagger} F$-Statistic.

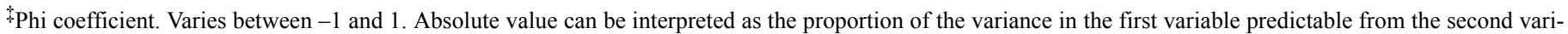
able (in this case, group membership).

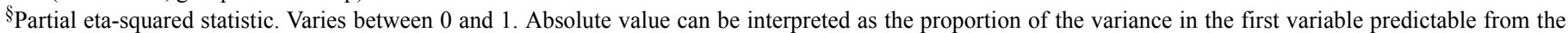
second variable (in this case, group membership).

"Averaged across right and left ear.

** Mean of dB HL thresholds at $0.5,1,2$, and $4 \mathrm{kHz}$.

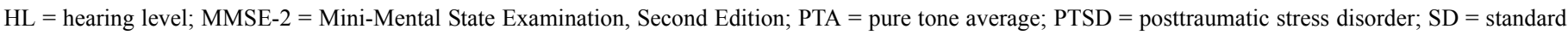
deviation; $\mathrm{SRT}=$ speech reception threshold; WRS $=$ word recognition score.

Table 2.

Details regarding blast exposure and mild traumatic brain injury (mTBI) diagnosis in the blast group.

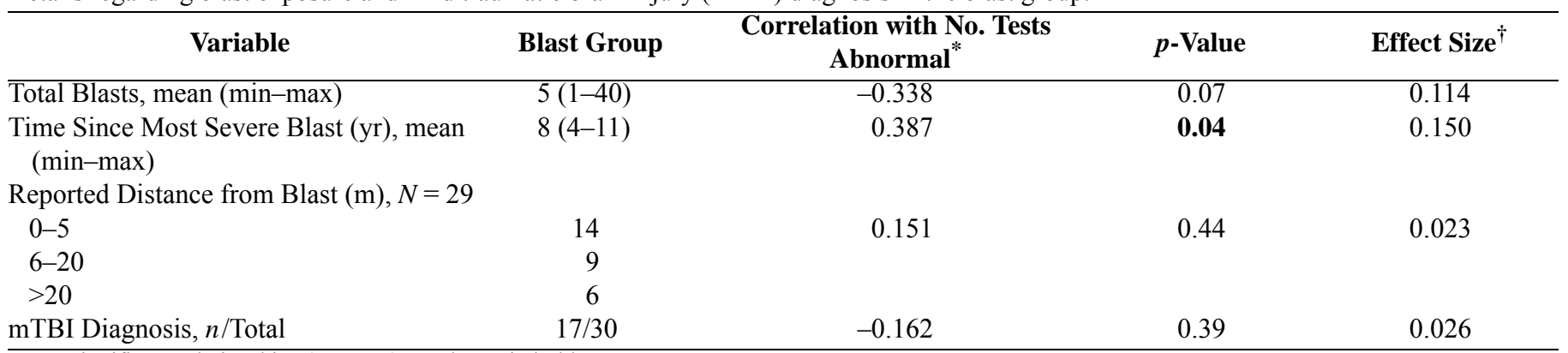

Note: Significant relationships $(p<0.05)$ are shown in bold.

*All correlations are Pearson correlations except the first row, Total Blasts, which was not normally distributed. In that case Spearman rho is reported.

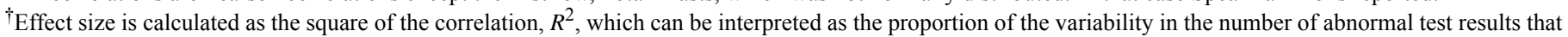
can be explained by each of the four variables.

$\max =$ maximum, $\min =$ minimum 


\section{Control Group}

The control group consisted of participants who did not have a history of blast exposure or other neurological injury. Throughout the initial contact and screening process, enrollment of this group was purposely delayed slightly relative to those who reported blast exposure. Every time several new members of the blast group had been enrolled, a few control group participants were contacted. Potential participants were contacted in the order in which they were received. In this way, recruitment was staggered such that the mean ages could be matched to those individuals in the blast group. Twenty-nine control participants without blast exposure or mTBI completed behavioral testing. As intended by design, all of the control participants fell in the same age range as the blast group. Initially, several female control group participants were tested, but it quickly became clear that the blast group would be overwhelmingly male and so enrollment of female control participants was halted to improve the sex balance. The ages and sex distributions of those included in this report are shown in Table 1.

\section{Intake Assessments}

The following measures were completed to ensure that all study participants met the inclusion criteria and to obtain information about potential covariates and details on subjective hearing abilities:

1. Audiometric Evaluation: This evaluation was conducted in a sound-treated booth using a calibrated audiometer and included pure-tone air- and boneconduction thresholds. Air-conduction thresholds were measured at frequencies up to $12.5 \mathrm{kHz}$. Speech reception thresholds (SRTs) were obtained in quiet using recorded Central Institute for the Deaf Spondaic Word
List W-1 spondaic words, and word recognition scores (WRSs) in quiet were obtained using recorded lists of 50 Northwestern University Auditory Test No. 6 monosyllabic words presented at $50 \mathrm{~dB}$ above each participant's measured SRT. Immittance audiometry, including tympanometry, ipsilateral and contralateral acoustic reflex thresholds, and contralateral acoustic reflex decay, were also completed. Means and standard deviations of audiometric evaluations, including the pure tone average (PTA) thresholds at $0.5,1,2$, and $4 \mathrm{kHz}, \mathrm{SRT}$, and WRS are shown in Table $\mathbf{1}$ for each participant group. In cases where these data were available from the National Center for Rehabilitative Auditory Research Data Repository within 6 mo of testing and the speech materials and procedures matched the study protocol, the repository data were used rather than repeating the tests.

2. Subjective Hearing Ability: The Hearing Handicap Inventory for Adults (HHIA) [5] was used to determine the degree to which hearing difficulties affected the lives of the participants. Scores on this test can be compared with published scores expected for participants with various ranges of hearing loss [6], and a similar metric has been shown to be sensitive to auditory processing difficulties [7-8]. Means and standard deviations of HHIA responses for each participant group are provided in Table 3.

3. Assessment of Cognitive Status: The Mini-Mental State Examination, Second Edition [9] evaluates gross cognitive dysfunction (i.e., dementia) on a variety of abilities: orientation to time and place, attention/concentration, language, constructional ability, and immediate and delayed recall. For this report, a score of $\geq 24$

Table 3.

Mean scores across groups on the Hearing Handicap Inventory for Adults (HHIA). In addition to mean group values, the percentage of individuals in each group classified as no handicap (scores $<17$ ), moderate handicap (scores 17-42), and severe handicap (scores $>42$ ) are provided.

\begin{tabular}{|c|c|c|c|c|c|}
\hline Variable & Control & Blast & Statistic & $p$-Value & Effect Size \\
\hline Average Score (mean \pm SD) & $2.7 \pm 8.9$ & $33.3 \pm 27.0$ & $33.01^{*}$ & $<0.001$ & $0.367^{\dagger}$ \\
\hline Handicap (\%) & & & $21.3^{+}$ & $<0.001$ & $0.601^{\S}$ \\
\hline None & 93 & 40 & & & \\
\hline Moderate & 7 & 23 & & & \\
\hline Severe & 0 & 37 & & & \\
\hline
\end{tabular}

${ }^{*} F$-Statistic.

†Partial eta-squared.

$\star^{2}$. The single $\chi^{2}$ value shown refers to the likelihood of obtaining the shown distribution of response across all three of the handicap levels if the two groups were not statistically different in terms of perceived handicap.

$\S_{\text {Cramer V. }}$ 
out of 30 was taken as indicating no evidence of gross cognitive dysfunction. None of the participants scored lower than 24, so all were included. Means and standard deviations for both groups are shown in Table 1.

4. Assessment of Blast Exposure: A revised version of the Warrior Administered Retrospective Casualty Assessment Tool (WARCAT) [10] was administered via computer, with help from the experimenter if needed. The WARCAT establishes the term of military service and collects detailed information from blastexposed individuals about their blast exposure (e.g., time since the blast exposure(s), proximity, and nature of the blast). For those exposed to multiple blasts, the relevant section of the WARCAT was filled out for each incident. Blast-related details obtained from the WARCAT are provided in Table 2.

5. Diagnoses of Traumatic Brain Injury and/or Posttraumatic Stress Disorder (PTSD): Patient medical records were searched following testing and were used to identify those who had a diagnosis of mTBI and/or PTSD. Participants were identified as having a medical diagnosis of mTBI when electronic health records available for the Northwest Veterans Integrated Service Network (VISN) 20 reflected one of the following International Classification of Diseases, Ninth Revision, Clinical Modification (ICD-9-CM) codes: 850.0 (concussion-no loss of consciousness), 850.9 (concussion-unspecified), 850.11 (concussion with loss of consciousness less than $30 \mathrm{~min}$ ), 854.0 (intracranial injury-unspecified loss of consciousness), 854.02 (intracranial injury with brief loss of consciousness), or V15.52 (history of traumatic brain injury). Participants were identified as having a medical diagnosis of PTSD only when VISN 20 health records reflected an ICD-9-CM code of 309.81. This particular designation refers to patients diagnosed with chronic PTSD and is distinct from diagnoses of acute stress disorders and posttraumatic brain syndrome. To provide a measure of PTSD severity, a four-item PTSD screening tool was administered. This tool has been shown to be strongly correlated with other more comprehensive measures [11].

\section{Inclusion Criteria}

All study participants met the following inclusion criteria: (1) PTA at $0.5,1,2$, and $4 \mathrm{kHz}$ of $35 \mathrm{~dB}$, i.e., normative hearing levels (HLs) or better; (2) no worse than $40 \mathrm{~dB}$ HL pure-tone hearing sensitivity at any two audiometric frequencies below $8 \mathrm{kHz}$; (3) no differences in hearing thresholds exceeding $10 \mathrm{~dB}$ HL between the two ears at more than one audiometric frequency below $4 \mathrm{kHz}$; (4) no evidence of conductive dysfunction, as indicated by bone conduction thresholds within $10 \mathrm{~dB}$ of air conduction thresholds at octave frequencies between 0.5 and $4 \mathrm{kHz}$; (5) native speaker of English; and (6) willing and able to give written, informed consent prior to participating in any of the study procedures. Once inclusion criteria were met, participants were placed in the "Blast" and "Control" groups based upon self-report of blast exposure as described previously. An additional group of participants, those with non-blastrelated $\mathrm{mTBI}$, are also being tested in the ongoing study. Data from this participant group will be addressed at a later date because insufficient numbers have been tested at this time.

\section{Behavioral Tests of Central Auditory Function}

Subjects were administered a variety of behavioral tests of central auditory function. For the purposes of this brief report, only the results of the tests that were included in the previous investigation [3] will be described to allow comparison between acute versus chronic results. Because all of these tests are fully described in the literature as well as in our previous report [3], only a summary of the most relevant features is included for each.

1. The Gaps-In-Noise (GIN) test [12] is a test of temporal acuity that evaluates the ability of a listener to detect brief silent intervals in ongoing bursts of broadband noise. Gap durations range from 2 to $20 \mathrm{~ms}$ in duration. The listener is instructed to press a response button every time a gap is detected. A short practice list containing only gaps ranging between 8 and $20 \mathrm{~ms}$ was presented to the right ear before testing started. Each ear was tested separately, with the right ear tested first. Scores are based on the percentage of correct responses at each gap duration, and the threshold estimate reflects the shortest duration at which (1) the listener scores greater than 50 percent correct (gaps detected on 4 of the 6 presentations) and (2) the listener scores greater than 50 percent on all long durations. As was done previously [3], the version of the test used in the published report [12] from which we took normative values was used rather than the slightly modified version of the test that is currently available. 
2. The Dichotic Digits Test (DDT) [13-14] consists of four numbers, two spoken to each ear, that the listener is required to repeat back to the tester. Order of report is not scored. Twenty items were presented, and listeners were encouraged to guess rather than failing to respond.

3. The Staggered Spondaic Words (SSW) test [15], a measure of dichotic listening, presents 40 pairs of "spondaic" words, each "spondee" consisting of two complete one syllable words (e.g., "cupcake") spoken with equal emphasis on both syllables. In each trial, the two spondees are presented, one to each ear, such that the first syllable of the first spondee is presented in quiet in one ear and the second syllable of the first spondee overlaps the first syllable of the second spondee in the opposite ear. Presenting to either the left or right ear first results in four test conditions: (1) right noncompeting, (2) right competing, (3) left noncompeting, and (4) left competing. Four practice trials were presented before testing started. Each syllable in each spondee was marked as correct or incorrect, and any reversals of word order were recorded but were not marked as incorrect. Scores for the four conditions, total number of errors, and number of reversals were all used as outcome measures. Listeners were not required to answer quickly, and breaks could be taken during the testing if desired.

4. The Frequency Patterns Test (FPT) [16], a test of temporal pattern perception, consists of sequences of three $200 \mathrm{~ms}$ tone bursts separated by $150 \mathrm{~ms}$ of silence. Two of the tones are the same frequency (either $880 \mathrm{~Hz}$ or $1,122 \mathrm{~Hz}$ ) and one tone is higher $(1,122 \mathrm{~Hz})$ or lower $(880 \mathrm{~Hz})$. Subjects were instructed to verbally repeat the words "high" for $1,122 \mathrm{~Hz}$ tones and "low" for the $880 \mathrm{~Hz}$ tones in a sequence that matched the tone sequence (e.g., "high high low"). They would have been allowed to hum or sing the sequence aloud, but none chose to do so. Subjects were given three practice sequences before beginning the test trials. Testing of the right ear preceded that of the left ear, and 15 items were presented to each ear. Any tone incorrectly labeled resulted in an incorrect trial.

5. The Masking Level Difference (MLD) test measures binaural processing. The version of the test [17-18] used in both the current and previous investigations [3] estimates thresholds for tones presented in a series of trials in which a noise burst is presented to both ears (in phase, or "diotically"). The signal to be detected consists of five $500 \mathrm{~Hz}$ tone bursts separated from each other by $250 \mathrm{~ms}$ of silence. On each trial, the set of tones is presented simultaneously with a $3 \mathrm{~s}$ noise burst with a bandwidth extending from 200 to $800 \mathrm{~Hz}$. Tone bursts are presented either in phase at the two ears (noise in phase, signal in phase $\left[\mathrm{N}_{0} \mathrm{~S}_{0}\right]$ ) or out of phase at the two ears (noise in phase, signal out of phase $\left.\left[\mathrm{N}_{0} \mathrm{~S}_{\pi}\right]\right)$ at a variety of signal-to-noise ratios, and occasional "catch trials" are interspersed in which only the noise stimulus is presented with no tones. Listeners verbally indicate whether or not a tone was present. Before testing, listeners were given practice on a set of three trials representing the three conditions, and testing was initiated once correct responses were elicited for all three stimuli. Scoring is based on the number of correct responses obtained for the two tone-present conditions (in phase and out of phase), with threshold estimated from the number of correct trials using an algorithm based on the Spearman-Kärber method [18] that is provided along with the test materials. The number correct for the noise-alone trials is computed with the intention that any participant who misses more than 2 of the 11 noise-alone trials is to be excluded from further analysis. None of the participants described in this report missed more than one, and therefore none were excluded based on this criterion. The MLD value in decibels is estimated by subtracting the $\mathrm{N}_{0} \mathrm{~S}_{\pi}$ threshold from the $\mathrm{N}_{0} \mathrm{~S}_{0}$ threshold.

While the same behavioral tests were administered here as in the previous study by Gallun et al. [3], there are marked differences in test administration that should be noted. Instead of using a CD player and written score sheets, as is common in clinical settings, computerized versions of the same tests were developed in which the audio from the CDs was triggered by a program written in MATLAB (MathWorks; Natick, Massachusetts) and played through a digital-to-analog converter and amplifier connected to the inputs of the clinical audiometer used to perform the audiometric evaluation. Scoring and storage of results were performed by the computer program, as was the randomization of the test order. Changing to this test administration reduced the risk of error and ensured greater data integrity. The sounds were conveyed to the listener seated in a sound-treated booth by ER3A headphones (Etymotic; Elk Grove Village, Illinois). All tests were conducted at a minimum of $35 \mathrm{~dB}$ sensation level above the SRT at each ear, as determined during the initial intake measure. This is a lower level 
than was used in the previous study [3] and had the benefit of ensuring that levels were not uncomfortable for any participants.

Participants were encouraged to take breaks, and testing was discontinued if fatigue or irritation were evident. Behavioral testing took approximately $2 \mathrm{~h}$ per test session and was often conducted over the course of two or three sessions, frequently interspersed with other less demanding tests, not reported here. All testing was overseen by licensed audiologists with multiple years of experience working in auditory research.

\section{Statistical Approach}

All statistical analyses were conducted using SPSS, version 22 (IBM; Armonk, New York). Statistical significance was set using a criterion of $p<0.05$, with "trends" identified as values of $p<0.10$. Significance levels were not corrected for multiple comparisons based on the recommendation of Keppel and Wickens [19], who argue that when there is a theoretical reason for testing multiple differences, the tests are not "post hoc" and thus no correction is appropriate. Since all of the tests conducted were chosen based on previous work, we believe that correcting for multiple comparisons is not warranted. Instead, in all cases effect sizes were also analyzed using partial eta-squared, $R^{2}$, phi, or Cramer $\mathrm{V}$ as appropriate given the statistical test being conducted. For all four measures, the absolute value varies between 0 and 1 and can be interpreted as the proportion of the variance in one variable that can be explained by another variable.

(a)

Frequency $(\mathrm{Hz})$

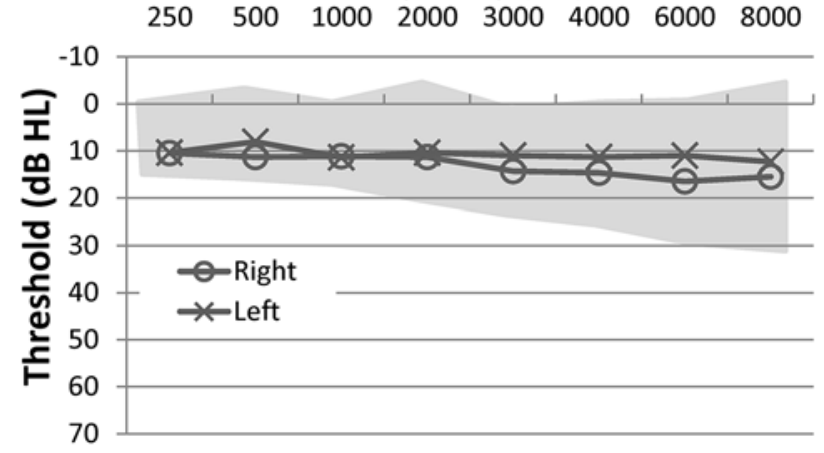

According to convention [19], effect sizes of 1 percent or greater are "small," 6 percent or above are "medium," and 15 percent or more (which corresponds to a correlation of just under 0.40) are a "large" amount of the variance. The criterion for assigning theoretical meaning to a difference between the groups was that it should both reach statistical significance and also capture a "large" proportion of the variance.

\section{RESULTS}

\section{Audiometric Evaluations}

Mean pure-tone air-conduction thresholds obtained from 0.25 to $8 \mathrm{kHz}$ for the right and left ears of the two groups are shown in Figure 1 along with \pm 1 standard deviation, indicated by shaded regions. The mean thresholds were all within the normal range through $8.0 \mathrm{kHz}$ ( $20 \mathrm{~dB}$ HL or better at all frequencies). The results for the ultrahigh frequencies of 10.0 and $12.5 \mathrm{kHz}$ are not shown in Figure 1 because not all participants were tested at the highest frequencies, but even in this range the mean thresholds were no greater than $30 \mathrm{~dB}$ HL among either the 23 blast-exposed or the 26 control participants who were tested. A repeated-measures analysis of variance (ANOVA) was conducted on the audiometric thresholds for left and right ears at the frequencies $0.5,1,2,4$, and $8 \mathrm{kHz}$ with group (blast-exposed vs control) as a between-subjects factor. Results indicated a significant difference between the groups $(F(1,57)=4.988, p=0.03$,

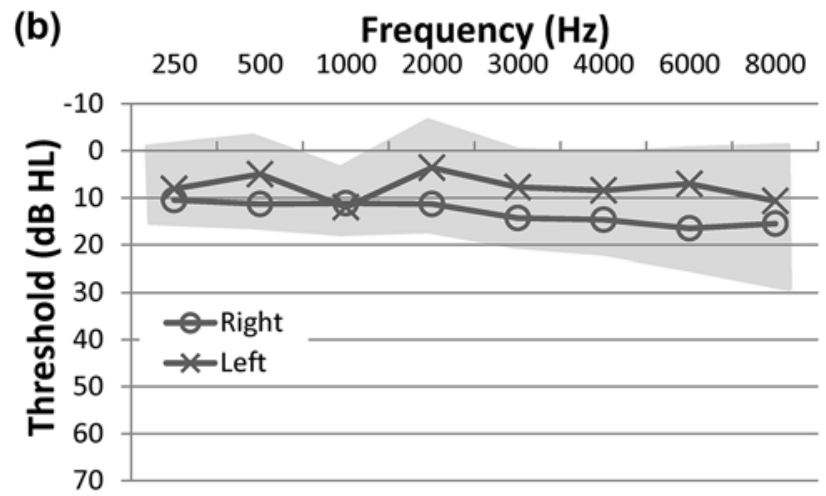

Figure 1.

Audiometric results for (a) blast-exposed group and (b) control group. Mean left (x) and right ear (o) thresholds are indicated for each of the octave frequencies between 0.25 and $8 \mathrm{kHz}$ for each group. Shaded areas indicate \pm 1 standard deviation of the mean. $\mathrm{HL}=$ hearing level. 
partial eta-squared $=0.03$ ), with the blast-exposed participants having group average thresholds between 2 and $6.4 \mathrm{~dB}$ poorer across frequencies than control participants. This effect size of 3 percent was small enough that this result was not considered theoretically meaningful. There were no significant interactions between ear, frequency, and group. Differences in both the PTA $(2.6 \mathrm{~dB})$ and the SRT ( $2 \mathrm{~dB})$ were also small, with effect sizes of less than 6 percent, as shown in Table 1. These scores show that on average speech understanding in quiet was within normal limits and similar for both groups.

\section{Subjective Hearing Ability}

Hearing complaints as indicated by scores on the HHIA [5-6] are shown in Table 3. Scores were substantially higher in the blast-exposed group, with 23.3 percent endorsing statements consistent with moderate hearing handicap (scores of 17-42) and 36.7 percent endorsing statements consistent with severe hearing handicap (scores $>42$ ) [6]. In contrast, only 6.9 percent of the control subjects endorsed statements consistent with moderate hearing handicap and none $(0 \%)$ endorsed statements consistent with severe handicap. A $t$-test revealed a significant difference in the means between the blast group and the control group, and the effect size of 0.367 is quite large. A nonparametric chi-square test of the association between group membership and degree of severity of the HIAA complaints, based on the three groups shown in Table 3, was significant and showed that group membership captured 60 percent of the variance in categorization.

\section{Blast Exposure}

Study participants who found reporting the information about their blast exposure emotionally too difficult were not required to do so. Of the 30 participants who reported blast exposure and who are included in the analyses reported here, all but one were able to provide information about the number of blasts experienced, distance from the blast, and time since the most serious event. These data are shown in Table 2, along with the number of blast-exposed participants with a diagnosis of mTBI. Though the mean number of blasts is 5.1, this number is inflated by one participant who reported 40 blasts. A more accurate representation of the distribution is given by the median value of reported blast exposures, which is 3.0. This is still an indication that our participants reported experiencing a substantial number of exposures.
The mean time since the most serious exposure was reported as $8.0 \mathrm{yr}$, and 48.3 percent of the blast-exposed participants reported being within $5 \mathrm{~m}$ of the blast. Among the blast-exposed group, 56.7 percent had a diagnosis of mTBI in their medical records. No individuals in the control group had any exposure to blasts nor any diagnoses indicating $\mathrm{mTBI}$.

\section{Behavioral Tests of Central Auditory Function}

Data obtained from both groups on all tests of central auditory function are shown in Table 4, in addition to results of repeated-measures ANOVA assessing the significance of group differences for each measure. Table 5 shows the percentage of each group that performed abnormally on the given measure. Abnormal performance was established based both on published normative data, the reference for which is provided with each test name, and with reference to the mean and standard deviation of the control group. For both metrics, abnormal performance is defined as performance more than two standard deviations worse than the mean of the relevant reference group. Figure 2 summarizes these results by showing in graphical form the percentage of participants from each group performing abnormally on any of the components of each of the tests with reference to the control group. The advantage of using the control group reference, as was done in our previous study [3], is that the proportion of abnormal performances is less influenced by differences in the age and hearing abilities of the blast-exposed group relative to the normative samples used in the published studies.

Summaries of performance on each of the behavioral tests are given here. Table 5 shows the counts of subjects with abnormal results and assesses the probability of such a distribution arising by chance using chi-square tests. The number of participants tested from each group is shown in Table 4. Note that several of the tests were not completed by all of the blast group participants, leading to occasional values based on 28 or 29 participants rather than 30. This was due to two of the participants moving out of state before completing all of the tests.

1. GIN: The mean performance for each group shown in Table 4 reveals that blast-exposed participants had poorer gap duration thresholds in both the left and right ears than control participants. Based on a univariate ANOVA, this group difference was significant for both ears. Both groups also demonstrated significantly better performance in the right ear than 
Table 4.

Results of behavioral tests of central auditory processing ability.

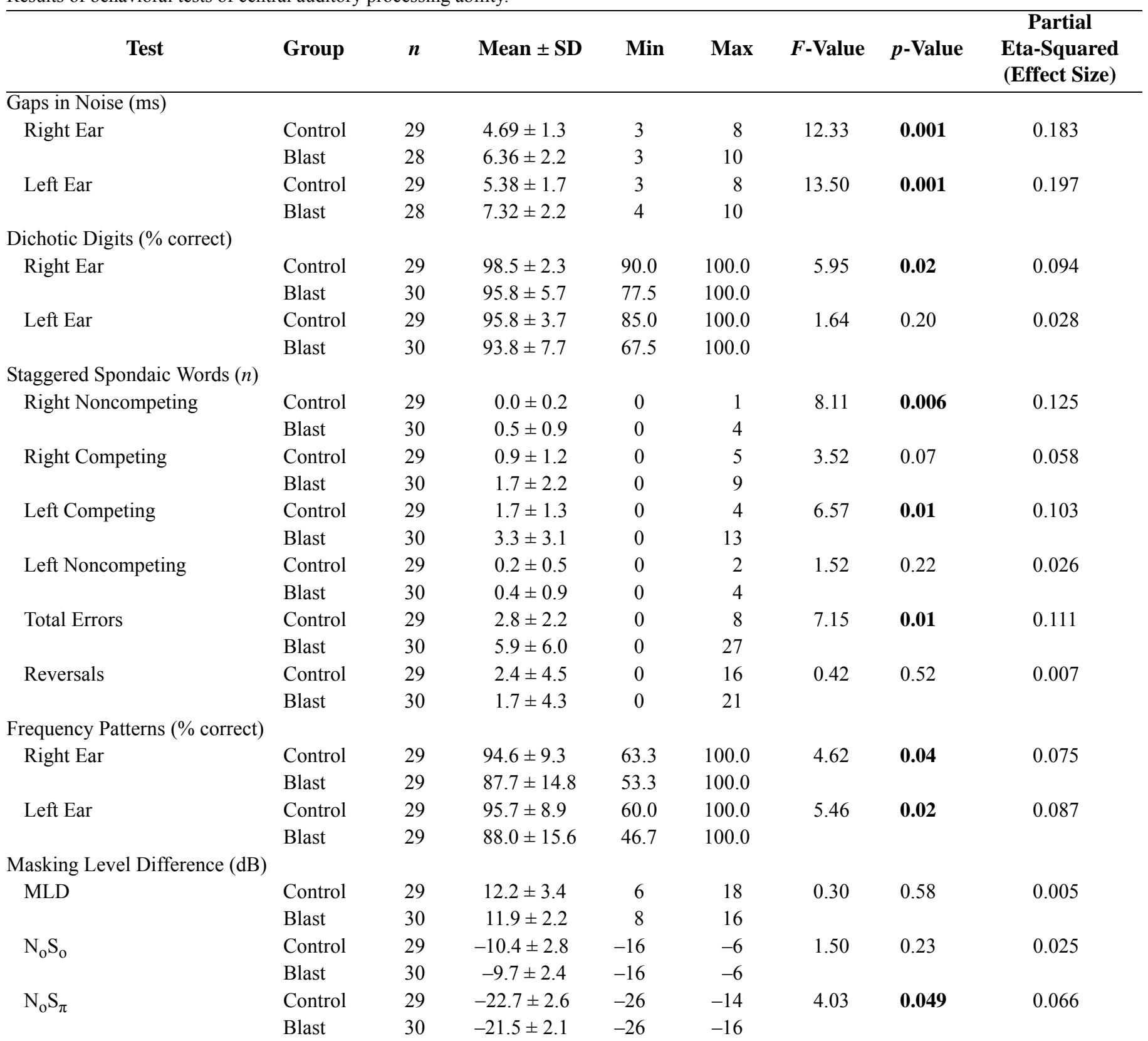

Note: $F$ - and $p$-values refer to analyses of variance conducted between the two groups for each of the components of the five behavioral tests. Bolded values indicate significant group differences $(p<0.05)$.

Max = maximum, Min = minimum, MLD = Masking Level Difference; $\mathrm{N}_{\mathrm{o}} \mathrm{S}_{\mathrm{o}}=$ noise in phase, signal in phase; $\mathrm{N}_{\mathrm{o}} \mathrm{S}_{\pi}=$ noise in phase, signal out of phase; $\mathrm{SD}=$ standard deviation.

in the left ear. The effect size of this difference was 0.183 for the right ear and 0.197 for the left ear, which suggests that it is both statistically significant and moderately large. Table 5 shows that the difference in the number of participants from each group performing abnormally using either of the two relevant criteria was statistically significant and that the effect sizes $(0.298$ and 0.418$)$ were between one and two times as large as effect size associated with the difference in the means. 
Table 5.

Counts of participants performing outside the normative range on behavioral tests of central auditory processing ability. Two normative ranges are reported: (1) the published values for groups of young listeners with normal hearing and (2) the values obtained with the control group of age- and hearing-matched participants tested in this study. For both sets of norms, "abnormal" is defined as performance values 2 standard deviations beyond the mean of the reference group. In cases where the ears were tested individually, an abnormal result at either ear was counted as abnormal performance.

\begin{tabular}{|c|c|c|c|c|c|c|c|c|c|c|}
\hline \multirow[b]{2}{*}{ Test } & \multicolumn{2}{|c|}{ Cutoff Value } & \multirow[b]{2}{*}{ Group } & \multirow[b]{2}{*}{$n$} & \multicolumn{2}{|c|}{ Abnormal (n) } & \multicolumn{2}{|c|}{ Chi-Squared } & \multicolumn{2}{|c|}{ Effect Size $^{*}$} \\
\hline & $\begin{array}{c}\text { Published } \\
\text { Norms }\end{array}$ & $\begin{array}{l}\text { Control } \\
\text { Group }\end{array}$ & & & $\begin{array}{c}\text { Published } \\
\text { Norm }\end{array}$ & $\begin{array}{c}\text { Control Group } \\
\text { Norm }\end{array}$ & $\begin{array}{l}\text { Published } \\
\text { Norm }\end{array}$ & $\begin{array}{c}\text { Control } \\
\text { Norm }\end{array}$ & $\begin{array}{c}\text { Published } \\
\text { Norms }\end{array}$ & $\begin{array}{l}\text { Control } \\
\text { Norms }\end{array}$ \\
\hline \multicolumn{11}{|l|}{ Gaps in Noise (ms) [12] } \\
\hline \multirow[t]{2}{*}{ Right Ear / Left Ear } & $6 / 6$ & $7.3 / 8.8$ & Control & 29 & 9 & 2 & 5.058 & 9.94 & 0.298 & 0.418 \\
\hline & & & Blast & 28 & 17 & 12 & & & & \\
\hline \multicolumn{11}{|l|}{ Dichotic Digits (\% correct) [14] } \\
\hline \multirow[t]{2}{*}{ Right Ear / Left Ear } & $90 / 90$ & $94 / 88$ & Control & 29 & 3 & 2 & 4.54 & 5.19 & 0.277 & 0.297 \\
\hline & & & Blast & 30 & 10 & 9 & & & & \\
\hline \multicolumn{11}{|l|}{ Staggered Spondaic Words (n) [15] } \\
\hline \multirow[t]{2}{*}{ Total Errors } & 6 & 7.15 & Control & 29 & 2 & 2 & 6.36 & 3.08 & 0.328 & 0.229 \\
\hline & & & Blast & 30 & 10 & 7 & & & & \\
\hline \multicolumn{11}{|l|}{ Frequency Patterns (\% correct) [16] } \\
\hline \multirow[t]{2}{*}{ Right Ear / Left Ear } & $75 / 75$ & $76.0 / 77.9$ & Control & 29 & 2 & 2 & 3.08 & 5.19 & 0.229 & 0.297 \\
\hline & & & Blast & 29 & 7 & 9 & & & & \\
\hline \multirow[t]{2}{*}{ Masking Level Difference (dB) [18] } & 8 & 5.5 & Control & 29 & 6 & 0 & 1.30 & $-\dagger$ & 0.149 & $-\dagger$ \\
\hline & & & Blast & 30 & 3 & 0 & & & & \\
\hline \multirow{2}{*}{\multicolumn{3}{|c|}{ Abnormal Performance on One or More Test (n) }} & Control & 29 & 12 & 6 & 7.61 & 7.59 & 0.359 & 0.359 \\
\hline & & & Blast & 30 & 23 & 19 & & & & \\
\hline
\end{tabular}

2. DDT: Blast-exposed participants were generally less accurate at identifying digits presented simultaneously to the two ears than were control participants. Singlefactor ANOVA revealed that this difference in performance was significant for the right ear, but not for the left (Table 4). The size of this significant effect was only medium, accounting for about 9 percent of the variance. When examined in terms of proportion abnormal, however, the difference was significant regardless of the reference distribution used, and group membership accounted for more than 25 percent of the variance according to Cramer V.

3. SSW: The blast-exposed group had a greater mean number of errors for all measures, including competing and noncompeting conditions at the left and right ears, total number of errors, and reversals (which are not counted as errors). Based upon a single-factor, between-subjects ANOVA, significant differences between the means of each group were found for right ear noncompeting words, left ear competing, and total errors with a trend toward significance observed for right ear competing words (Table 4). When compared with the cutoffs from either the published literature or the within-study control group, the groups were significantly different and the effect size was large: 0.328 for the published norms and 0.229 for the norms based on the control group.

4. FPT: Based on percentage of correctly identified pitch patterns, the blast-exposed group performed poorer in both the left and right ear than the control group. A univariate ANOVA confirmed a significant effect of group membership for presentations to both the left and right ears (Table 4). Table 5 shows that while there was no significant difference between the number in each group who performed abnormally relative to the published norms [16], using the norms of the control group led to a significant difference in proportion of abnormal performances. Nonetheless, the effect sizes are quite similar (0.229 and 0.297).

5. MLD: Both groups performed similarly with regard to thresholds obtained in the $\mathrm{N}_{0} \mathrm{~S}_{0}$ and MLD conditions according to a single-factor ANOVA. Thresholds measured during the $\mathrm{N}_{0} \mathrm{~S}_{\pi}$ condition demonstrated a 


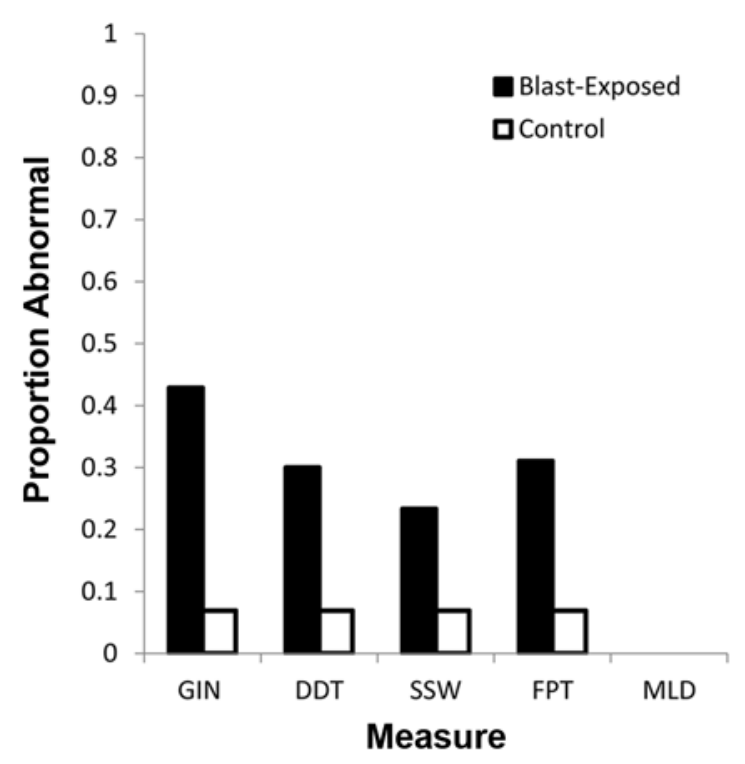

Figure 2.

Proportion of participants in blast-exposed group (filled bars) and control group (open bars) performing beyond range of normal performance for any of the components associated with each of five behavioral tests of auditory processing relative to the cutoff values based on the control group in the current study. DDT $=$ Dichotic Digits Test, FPT $=$ Frequency Patterns Test, GIN = Gaps-In-Noise, MLD = Masking Level Difference, SSW = Staggered Spondaic Words.

trend toward significantly poorer performance in the blast-exposed group based on single-factor ANOVA (Table 4). Relative to the published norms [18], which are only available for the MLD, the percentage of controls in the abnormal region (6/29) was greater than, but not statistically different from, the percentage of blast-exposed participants (3/30). This was just below the cutoff for a large effect size (Cramer $\mathrm{V}=$ 0.149). The number of participants performing abnormally in each group dropped to zero, however, when the means and standard deviations of the control group were used to establish within-study norms and when those values were used to examine the rates of abnormal performance on $\mathrm{N}_{0} \mathrm{~S}_{0}$ and $\mathrm{N}_{0} \mathrm{~S}_{\pi}$.

\section{Total Number of Tests in Abnormal Range}

Figure 3 shows the percentage of participants in each group for whom performance was abnormal on zero, one, two, three, or four of the five behavioral tests described previously when compared with the norms derived from the control group performance in this study. Table 5 shows that when compared with the published norms, 76.6 percent of the blast-exposed group was in the abnormal range on at least one test, as compared with 41.3 percent of the controls. Relative to the norms calculated based on the means and standard deviations of the control group in this study, 63.3 percent of the blast-exposed group were abnormal on one or more test, and 20.7 percent of the controls were in the abnormal range. The effect sizes of both comparisons were quite large: 0.359 . These percentages are quite similar to those in the previous report showing data for participants with more recent blasts exposures [3], in which 75 percent of the blastexposed participants were found to have abnormal performance on at least one test relative to the norms established by the age- and hearing-matched control group, in contrast to 24 percent of the control group.

\section{Potential Effects of Individual Factors}

Several variables are likely to affect performance on the present test battery, particularly those related to the extent of blast exposure. To assess these effects, correlations were analyzed between the total number of abnormal results on auditory processing tests (with reference to the control group distribution) and factors relating to blast severity including number of blast exposures, time

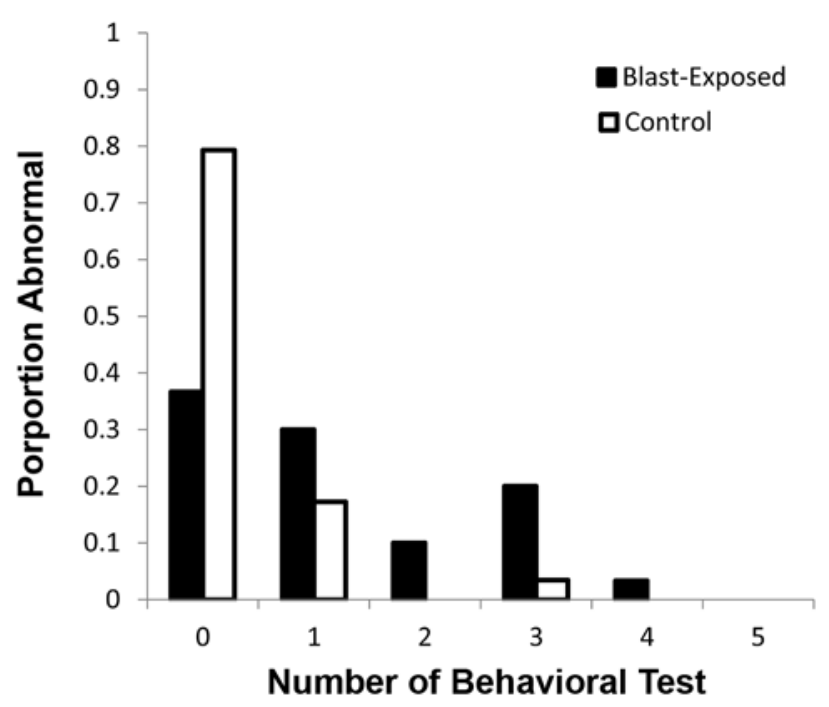

Figure 3.

Proportion of participants in blast-exposed group (filled bars) and control group (open bars) performing beyond range of normal performance for zero through five of the five behavioral tests of auditory processing relative to the within-study norms. 
since most severe blast exposure, distance from most severe blast, and diagnosis of mTBI. These results, shown in Table 2 , indicate that few physical indices of blast exposure correlate well with the number of abnormal results. Of all the blast-related indices, only time since blast was found to be significantly correlated, and the effect size was large, accounting for 15 percent of the variance in the number of tests with abnormal results. However, the correlation was in a somewhat unexpected direction in that those with longer times between blast exposure and testing tended to perform more poorly than those with more recent blast exposure. Further examination also revealed a significant positive correlation between participant age and time since blast exposure $(r=0.431, p=0.02)$ such that participants with longer time since blast exposure tended to be older. Partial correlations controlling for age revealed that the relationship between time since blast exposure and number of tests abnormal was no longer significant $(r=0.205, p=0.33)$.

To further assess the potential effects of participant age, we examined correlations between age and number of abnormal tests separately for the control and blast groups. In the control group, no significant relationship was found between age and total number of tests abnormal regardless of whether the published norms were used to define abnormal performance $(r=0.217, p=0.26)$ or whether abnormal was defined based on the distribution of the control group $(r=-0.093 ; p=0.63)$. The size of the effect of age was no greater than "small" for the control group (4\% of the variance explained or less than $1 \%$, respectively). For the blast group, aging was a statistically significant factor if the published norms were used ( $r=0.362, p=0.05)$ but not if the within-study norms were used $(r=0.305, p=0.10)$. In both cases, the effect of age was "medium" in size $(12.6 \%$ and $9.3 \%$ of the variance explained, respectively). Given that very little information is available regarding long-term effects of blast exposure, particularly with regard to the relationship with the natural aging process, these data may suggest that aging has a more deleterious effect on auditory processing in those who have been blast-exposed compared with those with no history of blast exposure. Thus, continuing to assess the effects of blast exposure and head injury throughout the aging process should be a high priority for future research.

Another relevant factor that may affect test results following head injury, according to the work of Lingma et al. [20], is PTSD. The number of participants in each group with a diagnosis of PTSD in their medical records is shown in Table 1. In the blast group, 77 percent had a diagnosis of PTSD, while only 14 percent had such a diagnosis in the control group. The mean values of the PTSD screening tool are also shown in Table 1. This instrument is composed of four questions related to PTSD symptoms, with possible responses ranging from 0 (not at all bothered) to 4 (extremely bothered). Thus, the total range of scores is between 0 and 16. Scores reported by the blast-exposed group were significantly higher than those reported in the control group, as revealed via $t$-test (Table 1). This difference is consistent with the higher rate of PTSD diagnoses in the blast group. The effect of PTSD on auditory processing tests was assessed by examining the correlations between PTSD diagnosis and PTSD "severity" (operationalized as the total score on the four-question screener), both of which were found to be correlated with the total number of abnormal tests relative to the within-study norms (severity: $r=0.307, p=$ 0.02; diagnosis: $r=0.292, p=0.03$ ). These effects were both in the medium range, accounting for 8 to 9 percent of the variance.

In an attempt to determine whether PTSD or blast exposure was more strongly related to abnormal performance in this sample, an ANOVA was conducted using number of abnormal tests (relative to within-study norms) as the dependent variable, group (Blast vs Control) as a between-subjects variable, and PTSD severity as a covariate. Severity was chosen over a PTSD diagnosis because it was a continuous rather than a dichotomous variable and thus would provide more information. The effect of group was statistically significant $(F(1,56)=5.20, p=0.03)$, indicating a significant difference in tests abnormal between the groups, while the effect of PTSD severity on abnormal test performance was not statistically significant $(F(1,56)=$ $0.123, p=0.73)$. The size of the group effect was estimated at 0.088 (medium) based on partial eta squared.

To further quantify the amount of variance on number of abnormal tests due to PTSD and blast exposure, linear regression models were fit with number of tests abnormal as the dependent variable and predictor variables of group, PTSD severity, and/or PTSD diagnosis. These models revealed that group membership alone accounted for 15.7 percent of the variance. The addition of PTSD severity as a predictor variable reduced the predictive value of the model to 14.3 percent, and the further addition of PTSD diagnosis as a predictor reduced the variance accounted for by the model to 13.3 percent. Examination of hearing loss, age, proximity to the blast, and TBI diagnosis as other potential covariates revealed 
that the only additional factor that significantly improved the prediction of the number of abnormal test results was age. A regression in which age and blast exposure were the only two factors included was able to account for 17.9 percent of the variance in the number of abnormal test results, with reference to the distribution of scores within the control group. These data suggest that age may be a mediating variable for the effects of blast exposure on auditory processing ability, but that PTSD does not appear to have the same effect.

\section{DISCUSSION}

Gallun et al. found that patients who had been exposed to high-intensity blast waves were more likely to show a reduced ability to process auditory information than were age-matched control subjects, even when peripheral hearing was near normal limits [3]. The data presented here replicate that finding, and in so doing show that reduced performance on tests of central auditory function relative to control participants can be present in individuals tested an average of more than $7 \mathrm{yr}$ after blast exposure. The persistence of this pattern of auditory dysfunction suggests that auditory processing ability does not recover within 4 yr (the minimum time since blast exposure in this study). Further, as these Veterans age, our data indicate that they are more likely to suffer from auditory processing deficits than their nonblast-exposed counterparts. These findings have direct clinical implications for audiologists and other clinicians treating Veterans exposed to blasts during their military service. Not only is an audiogram insufficient to evaluate the problems these individuals experience, but the inability to process auditory information has the potential to affect the care Veterans obtain, especially if these deficits are not recognized by the caregiver. Based upon responses on the HHIA, these patterns of dysfunction are causing difficulties in many environments where social interaction is necessary, and these difficulties are having significant effects on the emotional health of these Veterans. Unless the hearing health specialist is able to provide the appropriate diagnosis and deficit-specific counseling, the Veteran is left unaware of the reasons for his or her difficulties and other people in the Veteran's life will be unable to understand what is happening and adjust their behaviors appropriately.

The finding that an mTBI diagnosis was not strongly correlated with the number of abnormal test performances suggests either that the problems our participants are experiencing are not associated with brain injury or that the techniques used to identify mTBI in this population are not appropriate for identifying auditory dysfunction associated with mTBI. One alternative hypothesis is that PTSD was driving the auditory dysfunction by some as yet unknown mechanism. However, a PTSD diagnosis was not significantly related to auditory dysfunction once a report of blast exposure was taken into account. This raises the possibility that brain injury did occur, but that the mTBI diagnosis is based on factors that are not strongly related to the likelihood of experiencing auditory dysfunction. One of the complications of relying upon the mTBI diagnosis is that there are a number of difficulties inherent in determining both the presence and severity of mTBI, particularly in the Veteran population. In the case of active servicemembers, TBI diagnosis may not be made until several days, weeks, months, or years have passed, making it difficult to ascertain the presence and severity of the initial injury. This situation may lead to many servicemembers who are never accurately diagnosed with a brain injury. Furthermore, this study relied on proper ICD-9-CM code entry of any diagnosis made, which adds another potential source of error. It may be for this reason that group membership (Blast vs Control) was a better predictor of dysfunction than a diagnosis of mTBI despite the large number of members of the Blast group $(17 / 30)$ who had mTBI diagnoses.

The diagnosis of PTSD is less negatively affected by the time between blast exposure and clinical diagnosis because PTSD is a chronic disorder that can only be diagnosed after a significant amount of time has elapsed since the traumatic event. However, there are also reasons why the PTSD severity measure might not be as strong a predictor as blast exposure. PTSD severity is known to vary from day to day and is likely to be affected by various treatments prescribed by mental health professionals to study participants. We examined two measures of PTSD: both medical diagnosis and a measure of PTSD severity. These two measures were generally quite consistent, such that patients with diagnoses of PTSD had higher response scores on the PTSD severity measure (indicating more difficulties with symptoms often associated with PTSD) while those without a diagnosis had lower scores (indicating few difficulties).

The analyses showing the inability of PTSD severity to provide information about central auditory dysfunction are an essential finding of this study. While PTSD and TBI are often present in the same individuals, this correlation 
by itself does not imply that PTSD is primarily responsible for central auditory processing deficits. One alternative hypothesis that could explain some of the relationships observed here and in other studies between TBI and PTSD is that blast exposure can damage the brain in many ways, one of which may be to make an individual more likely to be diagnosed with PTSD [2122]. It would be beneficial if future studies are designed in a way that enables researchers to reveal the full set of relationships among TBI, PTSD, central auditory processing, sensory processing in general, and cognitive processes such as memory, attention, and executive function. In addition to further testing in these areas, future studies would benefit from attempts to combine these metrics with structural and functional imaging, as such studies have already shown the potential to help dissociate factors such as PTSD from mTBI [23].

Until such studies are conducted, it remains challenging to provide recommendations regarding best practices when it comes to diagnosis, counseling, and rehabilitation for these blast-exposed Veterans. The results of this study and the earlier study [3], however, suggest that Veterans with a history of blast exposure might experience deficits in processing of auditory information despite normal peripheral hearing. Assessment of central auditory processing should be considered as part of an interdisciplinary work-up on these individuals and the results considered in any rehabilitation plan.

\section{LIMITATIONS}

The results reported here represent one of only a handful of studies examining the relationship between blast exposure and auditory function. When a field of inquiry is as new as this one, it is essential to keep in mind the potential limitations of the studies and the many areas in which further research is needed. The most important limitation of this study is the relatively small sample size of the blast-exposed group. Now that it has been shown that significant differences on the same tests of auditory processing can be found in two independent samples of blast-exposed Veterans, additional follow-up studies with larger sample sizes would potentially provide additional information about these difficulties our Veterans are experiencing and reporting.

The current study is also limited in the sampling of participants, both with regard to sex and with regard to PTSD. The analyses reported here are limited by the lack of equal numbers of male and female participants in the control and blast groups, as well as the unequal distribution of those with and without PTSD of equal severity. This sampling issue should also be addressed in future studies.

Additionally, the trend toward significant differences in the peripheral hearing of the blast and control groups is a limitation. To better evaluate the importance of these and the other differences observed in this study, effect sizes were calculated for all of the measures discussed. The effect size for the relationship between number of abnormal test results (relative to controls) and the binaural PTA was 0.03 (3\% of the variance in number of abnormal tests was explained by PTA) and the effect size for the SRT was 0.02 for the right ear and 0.01 for the left ear, suggesting that these differences in audiometric thresholds are not strongly related to performance. Nonetheless, future studies would benefit from seeking to do a better job of equating audiometric values across participants.

Finally, it would be of great benefit in future studies to move beyond reliance upon ICD-9-CM codes for determining a TBI diagnosis.

\section{CONCLUSIONS}

Comparisons on behavioral auditory tests between subjects who have been exposed to high-intensity blasts at least 4 yr ago and those who report no blast exposure support the following conclusions.

Even though all subjects had normal or near-normal audiometric pure-tone thresholds and no evidence of peripheral auditory dysfunction, 60 percent of the blastexposed subjects reported hearing handicaps in the moderate to severe range on the HHIA, compared with only 7 percent of the control subjects scoring in the range of moderate handicap and none in the severe range. This result is entirely consistent with the complaints that audiologists hear in their clinics from military and Veteran patients who cannot hear everyday speech clearly despite normal performance on audiometric tests.

Sixty-three percent of blast-exposed group members $(19 / 30)$ showed abnormal performance on at least one of five tests of central auditory processing. Only 21 percent of the control group (6/29) performed abnormally on at least one test.

A statistical model including both age and blast exposure was the best predictor of the number of abnormal test results, suggesting the possibility that as individuals 
grow older the lasting effects of exposure to blasts may be exacerbated.

The central auditory tests that best differentiated the two groups involved temporal processing and tests of competing speech. This pattern of results is similar to the pattern observed in our previous work with patients who had been exposed more recently to blasts than those described here.

Many important questions remain concerning the relationship between blast exposure and auditory dysfunction. Although the severity of PTSD was not related to the number of subjects performing abnormally on auditory tests, more work is needed in this area to clarify the role of PTSD, the interactions with aging, and the similarities and/or differences between blast exposure (with or without a TBI diagnosis) and TBI from conventional causes such as sports-related concussion and motor vehicle accidents. In addition, future work should move beyond chart review and ICD-9-CM codes for identifying traumatic brain injury.

Finally, the mean performance of the members of the blast-exposed group was worse than the mean of the control group on every behavioral test of central auditory function, and in many cases, these differences were significant. This, along with our earlier report on subjects with more recent blast exposures, provides some support for the hypothesis articulated by the IOM report [1], that the auditory dysfunction observed in the acute stage of blast injury persists into the chronic stage.

\section{ACKNOWLEDGMENTS}

\section{Author Contributions:}

Study concept and design: F. J. Gallun, M. S. Lewis, R. L. Folmer, M. R. Leek.

Acquisition of data: M. Hutter, M. A. Papesh, H. Belding. Analysis and interpretation of data: F. J. Gallun, M. S. Lewis, R. L. Folmer, M. Hutter, M. A. Papesh, H. Belding, M. R. Leek. Drafting of manuscript: F. J. Gallun.

Critical revision of manuscript for important intellectual content: M. S. Lewis, R. L. Folmer, M. A. Papesh, H. Belding, M. R. Leek.

Financial Disclosures: The authors have declared that no competing interests exist.

Funding/Support: This material was based on work supported by the following awards from the VA Rehabilitation Research \& Development (RR\&D) Service: Merit Review Award C7755I (principal investigators: Gallun, Leek), Senior Research Career Scientist award to M.R. Leek (C4042L), and a Career Development II award to M. S. Lewis (C7067W). Support was also provided by the VA RR\&D National Center for Rehabilitative Auditory Research (C4844C).
Additional Contributions: Serena Dann, AuD; Daniel McDermott, MA; Tina Penman, AuD; ShienPei Silverman, MA; Emily Thielman, MS; Jay Vachhani, AuD; Leslie Grush, AuD; Ashley Hughes, AuD; Michelle Hungerford, AuD; and Kelly Kolonay also contributed to the success of this project. The authors gratefully acknowledge the enthusiastic participation of the Veterans and non-Veterans who volunteered for this study.

Institutional Review: The study was approved by the institutional review board at the VAPORHCS.

Participant Follow-Up: Participants were not offered follow-up information when giving informed consent to participate.

\section{REFERENCES}

1. Institute of Medicine. Gulf War and health: Long-term effects of blast exposures. Vol 9. Washington (DC): The National Academies Press; 2014.

2. Vanderploeg RD, Belanger HG, Horner RD, Spehar AM, Powell-Cope G, Luther SL, Scott SG. Health outcomes associated with military deployment: Mild traumatic brain injury, blast, trauma, and combat associations in the Florida National Guard. Arch Phys Med Rehabil. 2012;93(11): 1887-95. [PMID:22705240] http://dx.doi.org/10.1016/j.apmr.2012.05.024

3. Gallun FJ, Diedesch AC, Kubli LR, Walden TC, Folmer RL, Lewis MS, McDermott DJ, Fausti SA, Leek MR. Performance on tests of central auditory processing by individuals exposed to high-intensity blasts. J Rehabil Res Dev. 2012;49(7):1005-25. [PMID:23341276] http://dx.doi.org/10.1682/JRRD.2012.03.0038

4. Gallun FJ, Lewis MS, Folmer RL, Diedesch AC, Kubli LR, McDermott DJ, Walden TC, Fausti SA, Lew HL, Leek MR. Implications of blast exposure for central auditory function: A review. J Rehabil Res Dev. 2012;49(7):1059-74. [PMID:23341279] http://dx.doi.org/10.1682/JRRD.2010.09.0166

5. Newman CW, Weinstein BE, Jacobson GP, Hug GA. The Hearing Handicap Inventory for Adults: Psychometric adequacy and audiometric correlates. Ear Hear. 1990;11(6): 430-33. [PMID:2073976] http://dx.doi.org/10.1097/00003446-199012000-00004

6. Augustine AM, Chrysolyte SB, Thenmozhi K, Rupa V. Assessment of auditory and psychosocial handicap associated with unilateral hearing loss among Indian patients. Indian J Otolaryngol Head Neck Surg. 2013;65(2):120-25.

[PMID:24427551] http://dx.doi.org/10.1007/s12070-012-0586-6

7. Jerger J, Oliver TA, Pirozzolo F. Impact of central auditory processing disorder and cognitive deficit on the self-assessment of hearing handicap in the elderly. J Am Acad Audiol. 1990;1(2):75-80. [PMID:2132589] 
8. Fire KM, Lesner SA, Newman C. Hearing handicap as a function of central auditory abilities in the elderly. Am J Otol. 1991;12(2):105-8. [PMID:2053599]

9. Folstein M, Folstein S, White T, Messer M. Mini-mental state examination. 2nd ed. Lutz (FL): Psychological Assessment Resources; 2010.

10. Terrio H, Brenner LA, Ivins BJ, Cho JM, Helmick K, Schwab K, Scally K, Bretthauer R, Warden D. Traumatic brain injury screening: Preliminary findings in a US Army Brigade Combat Team. J Head Trauma Rehabil. 2009;24(1): 14-23. [PMID:19158592] http://dx.doi.org/10.1097/HTR.0b013e31819581d8

11. Bliese PD, Wright KM, Adler AB, Cabrera O, Castro CA, Hoge CW. Validating the primary care posttraumatic stress disorder screen and the posttraumatic stress disorder checklist with soldiers returning from combat. J Consult Clin Psychol. 2008;76(2):272-81. [PMID:18377123] http://dx.doi.org/10.1037/0022-006X.76.2.272

12. Musiek FE, Shinn JB, Jirsa R, Bamiou DE, Baran JA, Zaida E. GIN (Gaps-In-Noise) test performance in subjects with confirmed central auditory nervous system involvement. Ear Hear. 2005;26(6):608-18. [PMID:16377996] http://dx.doi.org/10.1097/01.aud.0000188069.80699.41

13. Broadbent DE. Perception and communication. New York (NY): Pergamon; 1958.

14. Musiek FE. Assessment of central auditory dysfunction: The dichotic digit test revisited. Ear Hear. 1983;4(2):79-83. [PMID:6840415] http://dx.doi.org/10.1097/00003446-198303000-00002

15. Katz J. The SSW test manual. 5th ed. Vancouver (Canada): Precision Acoustics; 1998.

16. Musiek FE, Pinheiro ML. Frequency patterns in cochlear, brainstem, and cerebral lesions. Audiology. 1987;26(2): 79-88. [PMID:3606474] http://dx.doi.org/10.3109/00206098709078409

17. Wilson RH, Moncrieff DW, Townsend EA, Pillion AL. Development of a $500-\mathrm{Hz}$ masking-level difference protocol for clinic use. J Am Acad Audiol. 2003;14(1):1-8. [PMID:12833923] http://dx.doi.org/10.3766/jaaa.14.1.2

18. Wilson RH, Weakley DG. The $500 \mathrm{~Hz}$ masking-level difference and word recognition in multitalker babble for 40to 89-year-old listeners with symmetrical sensorineural hearing loss. J Am Acad Audiol. 2005;16(6):367-82. [PMID:16178408] http://dx.doi.org/10.3766/jaaa.16.6.5

19. Keppel G, Wickens T. Design and analysis: A researcher's handbook. 4th ed. Upper Saddle River (NJ): Pearson Prentice Hall; 2004.
20. Lingsma HF, Yue JK, Maas AI, Steyerberg EW, Manley GT; TRACK-TBI Investigators. Outcome prediction after mild and complicated mild traumatic brain injury: External validation of existing models and identification of new predictors using the TRACK-TBI pilot study. J Neurotrauma. 2015;32(2):83-94. [PMID:25025611]

http://dx.doi.org/10.1089/neu.2014.3384

21. Eskridge SL, Macera CA, Galarneau MR, Holbrook TL, Woodruff SI, MacGregor AJ, Morton DJ, Shaffer RA. Influence of combat blast-related mild traumatic brain injury acute symptoms on mental health and service discharge outcomes. J Neurotrauma. 2013;30(16):1391-97.

[PMID:23489170]

http://dx.doi.org/10.1089/neu.2012.2537

22. Bazarian JJ, Donnelly K, Peterson DR, Warner GC, Zhu T, Zhong J. The relation between posttraumatic stress disorder and mild traumatic brain injury acquired during Operations Enduring Freedom and Iraqi Freedom. J Head Trauma Rehabil. 2013;28(1):1-12. [PMID:22647965] http://dx.doi.org/10.1097/HTR.0b013e318256d3d3

23. Petrie EC, Cross DJ, Yarnykh VL, Richards T, Martin NM, Pagulayan K, Hoff D, Hart K, Mayer C, Tarabochia M, Raskind MA, Minoshima S, Peskind ER. Neuroimaging, behavioral, and psychological sequelae of repetitive combined blast/impact mild traumatic brain injury in Iraq and Afghanistan war veterans. J Neurotrauma. 2014;31(5): 425-36. [PMID:24102309]

http://dx.doi.org/10.1089/neu.2013.2952

Submitted for publication December 19, 2014. Accepted in revised form December 4, 2015.

This article and any supplementary material should be cited as follows:

Gallun FJ, Lewis MS, Folmer RL, Hutter M, Papesh MA, Belding H, Leek MR. Chronic effects of exposure to high-intensity blasts: Results of tests of central auditory processing. J Rehabil Res Dev. 2016;53(6):705-20. http://dx.doi.org/10.1682/JRRD.2014.12.0313

ORCID: Frederick J. Gallun, PhD: 0000-0002-41452199; Melissa A. Papesh, PhD: 0000-0003-0853-3824

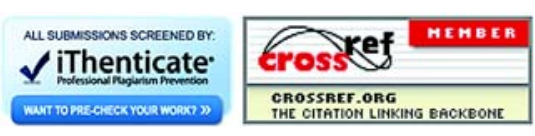

